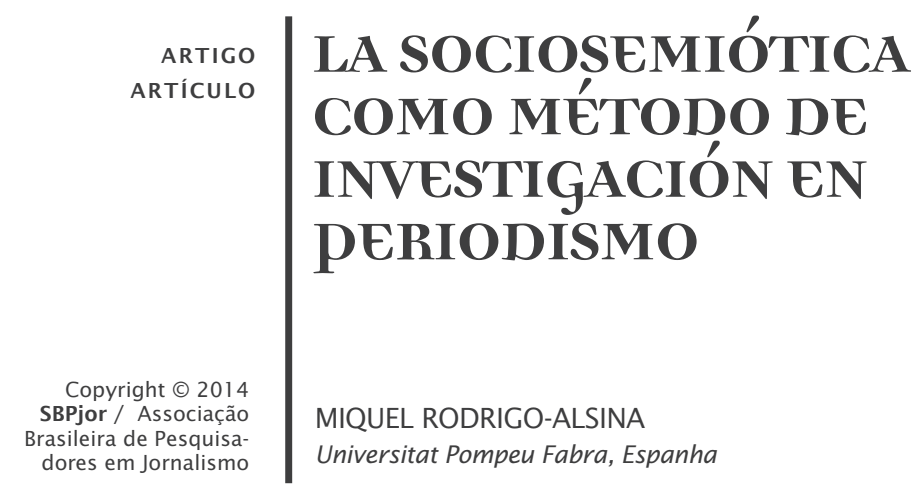

RESUMEN - Como se intenta mostrar en este artículo, la confrontación entre semiótica y sociología en los estudios de periodismo debe ser abordada a partir de los matices. En primer lugar, no es pertinente concebir un área de estudio como un campo de batalla en el que se enfrentan diferentes disciplinas. Por el contrario, existen muchos puntos en común entre sociología y semiótica. En segundo lugar, es necesario recordar que una de las controversias clásicas es la que opone el análisis cuantitativo, más característico de la sociología, al análisis cualitativo de la semiótica. Esta discrepancia no es nueva en las ciencias sociales (Alvira, 1983). Sin embargo, debemos señalar que nos encontramos ante una falsa dicotomía: los dos métodos no son incompatibles, sino complementarios. Palabras clave: Sociosemiótica. Periodismo.

\title{
A SÓCIO SEMIÓTICA COMO MÉTODO DE PESQUISA EM JORNALISMO
}

RESUMO - Como poderemos observar o confronto entre semiótica e sociologia nos estudos de jornalismo deve ser tomado a partir das nuances. Em primeiro lugar, não é pertinente conceber um campo de estudo como um campo de batalha, em que diferentes disciplinas confrontam-se. Ao contrário, existem muitos pontos em comum entre sociologia e semiótica. Em segundo lugar, devemos recordar que uma das mais clássicas controvérsias é a que opõe a análise quantitativa, mais característica da sociologia, versus a análise qualitativa, da semiótica. Esta oposição não é nova nas ciências sociais (Alvira, 1983). Entretanto, devemos pontuar que estamos em face a uma falsa dicotomia. Os dois métodos não são incompatíveis, mas sim complementares.

Palavras-chave: Sócio semiótica. Jornalismo

\section{SOCIO-SEMIOTICS AS A JOURNALISM RESEARCH METHOD}

\begin{abstract}
As it will be possible to observe, the confrontation between semiotics and sociology in the study of journalism should take on various shades of meaning. In the first place, it is not pertinent to conceive a field of study as a battlefield in which different disciplines confront each other. On the contrary, there are many points in common between sociology and semiotics. In the second place, we should recall that one of the classic counterviews is that of quantitative content analysis, more characteristic of sociology, versus qualitative semiotics analysis. This confrontation is not new in the social sciences (Alvira, 1983). However, we should point out that we find ourselves facing a false dichotomy. The two methods are not incompatible, but rather complementary. Keywords: Socio-semiotics. Journalism.
\end{abstract}


Lo primero que habría que plantearse es qué entendemos por sociosemiótica. Para ello, lo mejor es acudir a un clásico. En 1988, Hodge y Kress (1991:261) proponían la siguiente definición: "La semiótica social trata principalmente de la semiosis humana como un fenómeno social en relación a sus fuentes, funciones, contextos y efectos. También trata de los significados sociales construidos a partir de múltiples formas semióticas, a través de textos y prácticas, en todo tipo de sociedad humana y en todos los periodos de la historia humana." Como puede apreciarse estos autores hablan de "semiótica social", que es la terminología con la que en el ámbito anglosajón se refieren a la sociosemiótica.

Hagamos un poco de historia para explicar cómo se ha llegado a esta sociosemiótica. En 1973, Paolo Fabbri, en su conocido artículo de la revista Versus, "La communicazioni di masse in Italia: sguardo semiotico e malocchio de la sociologia", anunciaba la decadencia de la perspectiva sociológica frente a la semiótica en el estudio del periodismo. Para Fabbri la sociología tradicional entra en crisis al pasar de la ciencia de los hechos a la ciencia del sentido. Por ello, la semiótica es la disciplina más idónea para el estudio del periodismo. Pero quizás habría que matizar que hay distintas semióticas y distintas sociologías. Algunas son más adecuadas que otras para el estudio del periodismo. Además el objeto de estudio del sentido y de los discursos no es algo exclusivo de la semiótica. Como afirma Ferguson (2007: 52) "(...) el discurso y lo discursivo han llegado a ser conceptos clave en diversos ámbitos: el sociológico, el histórico, el académico, el de la investigación. Para quien estudia o investiga los medios es importante indagar cómo fue que el discurso y el discurso mediático han llegado a ser conceptos analíticos y teóricos fundamentales". Como veremos la aproximación semiótica inmanentista al periodismo, aunque es totalmente idónea de acuerdo con determinados objetivos de investigación, no es la única posible (VERÓN, 1987: 126-129; ABRIL, 2007: 96-104).

\section{NI SOCIOLOGÍA NI SEMIÓTICA O AMBAS: SOCIOSEMIÓTICA}

Como se podrá apreciar la confrontación entre la semiótica y la sociología en el estudio del periodismo debe matizarse. En primer lugar, no es pertinente concebir un campo de estudio como un campo de batalla en el que se enfrentan distintas disciplinas. Por el contrario, hay bastantes puntos en común entre la sociología y la semiótica. De hecho, como apunta Geertz (1976: 34), “la sociología del conocimiento debiera llamarse sociología del significado, pues 
lo que está socialmente determinado no es la naturaleza de la concepción, sino los vehículos de la concepción".

En segundo lugar, debemos recordar que una de las clásicas contraposiciones es la del análisis de contenido cuantitativo, más propio de la sociología, con el análisis semiótico cualitativo. Esta confrontación no es nueva en las ciencias sociales (ALVIRA, 1983). Sin embargo, deberíamos señalar que nos encontramos ante una falsa dicotomía. No son dos métodos incompatibles, sino complementarios. Obviamente, el análisis de contenido es un método más idóneo para corpus amplios (Glasgow Media Group, 1977 y 1980), mientras que la semiótica da lugar a estudios muy desarrollados de corpus reducidos (GREIMAS, 1976a).

Beltrán (1989: 33) toma una postura equidistante entre un "humanismo delirante", que rechaza una aproximación cuantitativa a los fenómenos humanos o sociales, y aquellos que desprecian cualquier aproximación que no sea cuantitativa y formalizable matemáticamente. La postura de Beltrán (1989: 40) es muy clara cuando señala lo siguiente:

no me interesa aquí establecer prelaciones, sino concurrencias; los métodos empíricos cuantitativo y cualitativo son, cada uno de ellos, necesarios in sua esfera, in suo ordine, para dar razón de aspectos, componentes o planos específicos del objeto de conocimiento. No sólo no se excluyen mutuamente, sino que se requieren y complementan, tanto más cuanto que el propósito de abarcar la totalidad del objeto sea más decidido.

\section{METODOLOGÍAS DE LA INVESTIGACIÓN EN PERIODISMO}

Hay que advertir que no todos los autores entienden lo mismo por metodología. Por mi parte voy a seguir la conceptualización que proponen Neuman (1994) o Del Rincón et al (1995) porque me va a permitir, por un lado, destacar otros problemas metodológicos y, por otro, recordar las fuentes de las teorías de la comunicación. Del Rincón et al (1995) proponen como tricotomía de los métodos de las ciencias sociales la metodología empírico-analítica, la constructivista y la sociocrítica. Neuman (1994), por su parte, utiliza la siguiente terminología al establecer su tricotomía: positivismo, ciencia social interpretativa y ciencia social crítica. Yo voy a adoptar la terminología: positivista, interpretativa y crítica.

Antes de entrar en cada una de las metodologías quisiera recordar que estas clasificaciones no han que entenderlas como barreras infranqueables, sino como un intento de organizar el conocimiento. Esto significa que, en ocasiones, entre las distintas metodologías hay puntos de conexiones y similitudes o las diferencias 
son simplemente un problema de la intensidad de la característica. Por ejemplo Neuman (1994: 69) señala que "El positivismo está basado en el determinismo: la conducta humana está determinada por leyes causales sobre las que sobre los seres humanos tienen poco control. La ciencia social interpretativa asume el voluntarismo: la gente tienen un amplio margen de libertad para crear los significados sociales. La aproximación de la ciencia crítica se sitúa en medio de ambas. Es parcialmente determinista y parcialmente voluntarista."

La metodología positivista es la metodología más próxima a las ciencias naturales. Así pues pretende establecer una serie de hipótesis que deben ser contrastadas de forma empírica. Pero no se trata sólo de verificar estas hipótesis para describir o explicar la realidad analizada, sino que hay una intención declaradamente prediccionista. Recordemos el aforismo positivista "saber para prever, prever para poder". Es decir, en última instancia, hay un deseo de predicción para poder controlar los fenómenos sociales. Orozco (1996: 32-33) diferencia el paradigma positivista del realista. Éste último, que es una variante del anterior, no pretende ser predictivo, pero si que considera que hay que llegar a las causas de los acontecimientos, a las explicaciones últimas. Aquí se encontrarían para Orozco (1996: 32) la mayoría de las investigaciones sobre los efectos de los medios de comunicación.

La metodología positivista corresponde a las ciencias nomotéticas, esto plantea el problema de si es aplicable a las ciencias sociales o sólo a las ciencias naturales. Para Orozco (1996:29) en las ciencias sociales "la explicación a los acontecimientos no está dada por el acontecimiento mismo, sino en el contexto, en el entorno en el cual se dan los acontecimientos. Esta es una de las tantas críticas que recibió el modelo positivista de parte de las ciencias sociales, cuando se trasladó el modelo de las ciencias físicas al modelo de las ciencias sociales". Otra de las críticas al positivismo es que contempla la "realidad de forma fragmentada y al centrarse en los fenómenos observables de la realidad corre el peligro de ignorar otras dimensiones de la misma. Por otro lado, algunas situaciones sociales son difíciles de observar sin ser distorsionadas, y algunos estudios pueden ser irrepetibles o de difícil replicación" (DEL RINCÓN et al, 1995: 28-29). Pero, por otro lado, se considera que es el único método realmente científico. Este método defiende la objetividad metodológica por lo que exige técnicas de investigación que sean independientes del investigador y que permitan la replicación. El positivismo considera 
que el único conocimiento válido es el que es verificable y medible. La cuantificación es básica para la metodología positivista. Para el positivismo las ciencias sociales son "un método que combina la lógica deductiva con las observaciones empíricas precisas de la conducta individual para descubrir y confirmar una serie de leyes probabilísticas causales que se pueden usar para predecir los modelos generales de la actividad humana" (NEUMAN 1994: 58).

Dentro de las teorías de la comunicación situaríamos en la metodología positivista a la perspectiva estructural funcionalista. Las técnicas usuales de la metodología positivista son los tests, los estudios de laboratorio, las encuestas, la observación sistemática y el análisis de contenido.

La metodología interpretativa, que en ocasiones se denomina hermenéutica, está fundada en las humanidades, aunque también hay que tener en cuenta a la sociofenomenología, a la semiótica y a la sociosemiótica. La metodología interpretativa busca descubrir los significados de las acciones sociales, sus prácticas y sus discursos. Es decir, no es tan importante lo que es un acontecimiento en sí mismo, como aquello que interpretan los actores sociales que es. Como señala Orozco (1996: 33) no se trata tanto de llegar a un conocimiento objetivo sino más bien a un conocimiento consensuado. Con la metodología interpretativa nos encontramos con

un proceso de investigación holístico-inductivo-ideográfico, buscando una comprensión global de los fenómenos y situaciones que estudia. Utiliza la vía inductiva, los conceptos, la comprensión de la realidad y las interpretaciones se elaboran a partir de la información. Se crea un clima social adecuado para que las personas puedan responder fielmente según sus experiencias y vivencias, teniendo en cuenta la idiosincrasia de los fenómenos y el contexto de las situaciones (DEL RINCÓN et al., 1995: 29-30).

Las críticas que se le suele hacer a esta metodología es su carácter subjetivo. Se afirma que el sistema de recoger la información es poco fiable porque los sujetos pueden dar datos incompletos o que el investigador puede dar una visión sesgada de la realidad. Aunque la generalización no es uno de los objetivos de la metodología interpretativa, el positivismo considera que lo particular no hace ciencia, por consiguiente cuestiona la cientificidad de esta metodología.

Para Neuman (1994: 62) "la aproximación interpretativa es el análisis sistemático del significado de la acción social a través de la observación directa de la gente en su espacio natural para llegar a entender e interpretar como la gente crea y mantienen sus mundos sociales". 
Dentro de las teorías de la comunicación por lo que respecta a la metodología interpretativa podríamos incluir a la Escuela de Palo Alto, al interaccionismo simbólico, al construccionismo y a la etnometodología. Las técnicas más utilizadas por la metodología interpretativa son la observación participante, los estudios de laboratorio, las historias de vida, las entrevistas en profundidad y el análisis discursivo.

La metodología crítica es básicamente una reflexión racional que busca desvelar la distorsión que la ideología, entendida como falsa conciencia, produce en la concepción de la realidad de las personas. Se pretende ampliar la conciencia crítica de las personas porque las ideologías dominantes bajo una apariencia de racionalidad ocultan unos intereses políticos particulares. Se trata de poner de manifiesto estas contradicciones y denunciar la apariencia de racionalidad que las ampara. Para la metodología crítica "la ciencia social es un proceso de análisis crítico que debe ir más allá de las ilusiones superficiales que ocultan las estructuras reales del mundo material para ayudar a la gente a cambiar las condiciones y construir ellos mismo un mundo mejor" (Neuman 1994: 67).

Las críticas que se suelen hacer a esta metodología son que está orientada políticamente, que se fundamenta en unos determinados valores y que es decididamente intervencionista en la realidad social. Todo ello le hace perder objetividad y neutralidad.

En la metodología crítica, con relación a las teorías de la comunicación, se podría recoger la Escuela de Francfort, los estudios de economía política, los estudios culturales y también la sociosemiótica. Aunque hay que señalar que los estudios culturales y la sociosemiótica también podrían enmarcarse en la metodología interpretativa.

Las técnicas usuales de la metodología crítica son la observación de la realidad social, las historias de vida, las entrevistas en profundidad y el análisis discursivo.

Para algunos autores "La ciencia crítica incorpora las prácticas y fines de ambas metodologías, la empírico-analítica y la constructivista, y conjuga relatos empíricos e interpretativos para facilitar sus fines dialécticos y críticos. La ciencia crítica busca recuperar el papel del teórico para la teoría social y la política en general"(DEL RINCÓN et al, 1995: 31 ).

Una de las clásicas discusiones entre las distintas metodologías son los criterios de rigor que utilizan. Como recogen algunos autores (DEL RINCÓN et al, 1995: 32-35) los criterios regulativos del rigor son 
la veracidad, la aplicabilidad, la consistencia y la neutralidad.

El criterio de veracidad hace referencia al grado de confianza que ostentan los procedimientos y los resultados de la investigación. El criterio de veracidad de la metodología positivista es interno. Es decir, que las variaciones que el investigador introduzca en las variables independientes sean la únicas causas de las variaciones observadas en la variables dependientes. Por lo que respecta a los resultados la veracidad se refiere al grado de correspondencia que existe entre los resultados obtenidos y la realidad analizada, que se considera que es única y uniforme. En las metodologías interpretativa y crítica "para conseguir la veracidad se recurre al criterio de credibilidad - paralelo al de validez interna -, que se recurre al contrastar distintas fuentes de información, a través del diálogo y de la argumentación racional, de procesos de 'corroboración estructural' y 'adecuación referencial', contextualizando la situación” (DEL RINCÓN et al, 1995: 33).

El criterio de aplicabilidad se refiere a si se pueden generalizar los resultados de la investigación. Mientras que en el positivismo la generalización es esencial, las otras dos metodologías relativizan su importancia. Sobre todo la metodología interpretativa se plantea hasta qué punto existen situaciones comparables, ya que el contexto y las circunstancias cambian con gran rapidez. Por ello se prefiere hablar transferibilidad a otros contextos muy similares. Con la metodología positivista la generalización se puede cuestionar si se pretende extrapolar los resultados de experimentos de laboratorio a la vida cotidiana. En cualquier caso, para conseguir la mayor generalización se buscará que la situación de investigación sea lo más representativa posible.

El criterio de consistencia o estabilidad hace referencia al grado de replicabilidad de la investigación. Esta estabilidad de los resultados es, para la metodología positivista, lo que da la fiabilidad a la investigación. En el caso de las otras metodologías no se plantean esta posibilidad de replicación, pero si que se lleguen a los mismos resultados a partir de interpretar las mismas informaciones con perspectivas similares.

El criterio de neutralidad apunta que la investigación no puede ver sus resultados condicionados por los sesgos, juicios o prejuicios e intereses del investigador. Para el positivismo la neutralidad se alcanza mediante la objetividad. En la metodología crítica se considera que el investigador siempre toma partido, aunque en las otras metodologías se niegue. La metodología interpretativa se basa en criterios intersubjetivos que, mediante la confirmabilidad, permiten detectar los sesgos personales de investigador. 
Por último, desearía apuntar que el estudio de la comunicación de masas, en general, y del periodismo, en particular, ha postulado mayoritariamente por una perspectiva pluridisiciplinar o/e interdisciplinar (RODRICO: 2001). Por ello es absolutamente pertinente que la base de las futuras investigaciones en este campo sea la sociosemiótica, ya que en ella se sintetizan las dos corrientes fundamentales, semiótica y sociología, aunque debamos tener también en cuenta las aportaciones de la psicología, e incluso de la antropología. En relación a la tricotomía que hemos recogido, en mi opinión, la sociosemiótica se situaría entre la interpretativa y la crítica, fundamentalmente, pero sin desdeñar tampoco el positivismo, o como minimo el realismo. En este sentido sería una metodología transversal, aunque, como veremos a continuación, hay distintas sociosemióticas.

\section{LAS SOCIOSEMIÓTICAS}

Desde el campo de determinada semiótica se ha reconocido, históricamente, una cierta autonomía de la sociosemiótica. Sin embargo, no parece haber mucho acuerdo sobre qué es o debería ser la sociosemiótica. Podemos distinguir básicamente dos posturas. En primer lugar, nos encontramos con una sociosemiótica unidisciplinar, dependiente de los criterios metodológicos de la semiótica discursiva. Esta sociosemiótica unidisciplinar tiene como objeto de estudios los discursos de lo social. En segundo lugar, tendríamos una sociosemiótica pluridisciplinar que reivindica una máxima autonomía a partir de la intersección de diferentes ciencias y metodologías en un objeto de análisis común. Este objeto podría ser, por ejemplo, el proceso de la construcción de la noticia (Rodrigo 2005). De todas formas también hay que recordar que "...no es ni posible ni conveniente aplicar la semiótica social o cualquier modo de análisis del discurso con metodologías mecánicas e inmutables" (FERGUSON 2007: 74). En primer lugar, una investigación no está al servicio de una metodología sino a la inversa es la metodología la que debe adecuarse a la investigación. Así, es el propio objeto de estudio y los objetivos de la investigación los que van a determinar cuál es la metodología más idónea. Un estudio global del discurso de la noticia requiere no sólo el analisis de sus estructuras narrativas sino también de su producción y de su comprensión (VAN DIJK, 1990). En segundo lugar, la investigación también puede hacer sus propuestas metodológicas de forma que en ella no sólo se describe y se explica un fenómeno comunicativo, sino que también se aporta a 
la comunidad científica una forma algo distinta de abordarlo.

La sociosemiótica unidisciplinar estaría representada, principalmente, por la ortodoxia de la Escuela Semiótica de Paris (Greimas y Courtés, 1982 y 1986). Esta sociosemiótica puede calificarse como una sociosemiótica del enunciado. Las fuentes de las que parte son la etnoliteratura $y$, fundamentalmente, la sociolingüística. Su finalidad es el establecimiento de una sociosemiótica que dé cuenta de las connotaciones sociales. Algunas de las dimensiones de este vasto ámbito son para Greimas y Courtés (1982: 391-394), las siguientes:

a) Una concepción del orden discursivo de una sociedad determinada (Foucault, 1978).

b) El establecimiento del estatuto veridictorio de los discursos en cada sociedad. Es decir, lo que se considera una historia "real" y una historia de "ficción".

c) La determinación de sociolectos y de los grupos sociosemióticos que los utilizan.

d) El reconocimiento y la organización de los discursos sociales: westerns, partidos de fútbol, danza, etc.

El objeto empírico de la sociosemiótica es definido por Landowski en los siguientes términos:

(...) el conjunto de discursos y de prácticas que intervienen en la constitución $\mathrm{y} / \mathrm{o}$ en la transformación de las condiciones de interacción entre sujetos (individuales o colectivos). Inicialmente centrada en el estudio de sistemas (taxonomía de lenguajes sociales, sistemas de connotaciones sociales), la problemática se reorienta así, poco a poco - a partir de la gramática narrativa -, hacia un mejor conocimiento de los procesos sociosemióticos (GREIMAS y COURTÉS, 1986: 207).

Las relaciones de esta semiótica unidisciplinar con la sociología son, por una parte, claramente distantes. Se prefiere la "coherencia metodológica" a las "ambiciones interdisciplinarias" (GREIMAS y COURTÉS, 1982: 392). La sociosemiótica depende, desde este punto de vista, del desarrollo de la semiótica general. Como apunta Landowski (1986: 303):

Si la sociosemiótica tiene, por ejemplo, algo que decir hoy (por poco que sea) sobre estrategias (...), si ambiciona incluso reformular ciertos conceptos claves de la sociología - "autoridad", "legitimidad", "poder", por ejemplo - es porque la semiótica general le suministra previamente algunos instrumentos operativos indispensables, que tienen el nombre, muy precisamente, de semiótica de la persuasión (hacer creer), semiótica de la acción (hacer ser), semiótica de la manipulación (hacer hacer) y sobre los cuales se injerta finalmente la semiótica de las pasiones (...). 
Pero, por otra parte, la sociosemiótica unidisciplinar también es beligerante con la sociología. Por ejemplo, considerar que no se puede decir que el análisis del enunciado no aclare mejor la naturaleza de la enunciación que "los parámetros sociológicos, independientemente de las substancias, canales o media que sirven para sus manifestaciones (televisión, cine, espectáculos de deportes colectivos, etc.), por el hecho de que todos remiten a un mismo universo significante y porque las formas de organización discursiva que se descubren en ellos son comparables." El propio Greimas (1976b: 58-59) apunta como características de este tipo de discursos, por un lado, la desaparición de las instancias de la enunciación o la aparición de un sujeto de la enunciación colectivo. Por otro lado, los textos sociales establecen explícitamente el modo de empleo para la lectura correcta de los mismos. Además no sólo hay una redundancia en los contenidos, sino también una recurrencia de las formas.

Si la sociosemiótica se limita al estudio de los discursos sociales, incluso en el sentido amplio del término, creo que, efectivamente, la sociosemiótica unidisciplinar es una disciplina idónea. Pero si pretende ir más allá del texto, se ha de plantear a partir de otros principios.

Ferguson (2007:57) señala: "La semiótica ha sido definida como la ciencia de los signos, y es sin duda una buena manera de analizar los mensajes mediáticos. Pero para familiarizarnos con esta importante herramienta analítica y filosófica es preciso que estudiemos otras variables básicas. Ya he dicho que el signo es una relación: una relación entre el significante y lo significado. Para que esa relación se establezca, tiene que haber alguien que perciba el signo. Ese alguien puedo ser yo, tanto si soy un miembro de una audiencia mediática como si estoy dirigiéndome a ella". En este mismo sentido, Hodge y Kress (1991: 12) afirman:

La semiótica tradicional asume que los significados relevantes
están inmóviles y fijos en el propio texto, para que sean
extraidos y descodificados por el analista a partir de un sistema
de codificación que es impersonal y neutral, y universal para los
usuarios del código. La semiótica social no puede asumir que
los textos produzcan exactamente los significados y los efectos
que sus autores esperan: así pues las confrontaciones y sus
consecuencias inciertas son las que deben ser estudiadas a partir
de la acción social, y sus efectos en la producción de significados.

Esta idea de que hay un significado profundo que el semiótico puede desentrañar parte de la concepción de un texto con una 
correspondencia unívoca, cada signo tendría así un sólo significado. Pero, como muy bien apunta Abril (2007: 26),

\begin{abstract}
El sentido es fluido, y aunque algunos discursos parecen cerrar regularmente sus posibilidades, el texto normalmente funciona como un sistema de significación multiestructurado, que se mueve de nivel en nivel, de forma que sus denotaciones se hacen connotaciones en progresión infinita. Así nunca se llega a una lectura final. La lectura de una semiótica crítica nunca llega a ser repleta ni cumplida, ni pretende descubrir sentidos ocultos y sacarlos a la superficie. Más bien trata de actuar con cierto rigor y complejidad, comprender la configuración y estructura de los textos y mantener la atención respecto a las relaciones de poder involucrados en ellos...
\end{abstract}

Si aceptamos que los significados son negociado socialmente y que pueden dar lugar a una agonística discursiva en que unos significados pretendan imponerse sobre otros (o mejor dicho que determinadas comunidades interpretativas intenten imponer un significado hegemónico), aceptaremos, con los anteriores autores, que un significante no siempre remite a un mismo sistema de significación. "Los cambios en su significado dependen de los diversos contextos sociales e históricos y de los diferentes discursos que situaron ese significante en determinadas maneras muy particulares" (FERGUSON, 2007: 60).

En esta línea, Abril (2007:26-27) apunta que hay que tener en cuenta - este autor se refiere a los textos visuales pero sería extrapolable a los textos escritos - las condiciones históricas culturales de producción, distribución y consumo-recepción de los textos de la siguiente forma:

a) "En primer lugar, leerlos contextualmente, es decir, interpretarlos en el marco de las instituciones, prácticas, modelos textuales y entornos técnicos en que son objetivados e intercambiados" (Abril, 2007: 26).

b) "En segundo lugar, interpretarlos reflexivamente, es decir por referencia a los efectos que, en tanto que prácticas textuales, producen sobre su propio contexto. Y aún más, teniendo presente que, sea cual fuere nuestra perspectiva, también ella tendrá una carácter contextual y reflexivo, y por tanto histórico - culturalmente determinado" (Abril, 2007: 27).

c) "En tercer, interpretar el texto discursivamente, como producido por un sujeto (individual o colectivo, autorreferente o no, mejor o peor identificado) que en él actúa y a la vez se constituye como agencia enunciativa en unas determinadas coordenadas espacio- 
temporales y en relación a reales o virtuales agencias enunciatarias (destinatarios)" (Abril, 2007: 27).

En mi opinión, en esta línea, hay dos conceptos fundamentales, y que están muy relacionados, para sociosemiótica del discurso periodístico. Uno de ellos es el de polisemia y el otro es el de intertextualidad.

En relación a la polisemia recordemos con Ferguson (2007: 73) que "Si en los medios el significado está ligado a la posibilidad de la polisemia, ello se debe en gran parte a los contextos sociales en los que el mensaje es recibido y luego decodificado o interpretado, y también a quien está haciendo la interpretación. Los textos se mantienen obstinadamente idénticos, mientras que sus significados pueden experimentar deslizamientos y cambios a lo largo del tiempo".

Por lo que hace a la intertextualidad hay que recordar que los textos son leídos a partir de su relación con otros textos. Recordemos que la primera actividad que lleva a cabo un usuario/receptor de un medio de comunicación es la interpretación que hace del discurso mediático. Dicha interpretación la hace desde su conocimiento, su experiencia, su historia de vida, su sentimentalidad, etc. Además esta interpretación puede dar lugar a interacciones intertextuales y extratextuales. Las interacciones intertextuales se producen porque los textos que los individuos hemos leído e interpretado dialogan entre sí. En las noticias en desarrollo, por ejemplo, es muy claro porque entre los distintos acontecimientos se van estableciendo relaciones intertextuales. Aunque también dialogan con otros relatos que la persona recuerde.

Las interacciones extratextuales estarían en relación con lo que Orozco (2001: 44-46) denomina interacciones de segundo orden en la que la interacción comunicativa no se produce con el medio de comunicación sino con otras personas, pero sobre, por ejemplo, el relato televisivo - de ahí que las denominemos "extratextuales" porque van más allá del texto televisivo propiamente dicho.

Esto nos lleva de nuevo al otro concepto, en la línea de lo que estamos explicando, que es el de polisemia. No se trata que un discurso tenga una intención polisémica, sino que desde el punto de vista de la interpretación todos los discursos se vuelven polisémicos.

Como señala Orozco (2006:23):

Los ER [Estudios de Recepción] han asumido la 'polisemia' como característica o cualidad de cualquier referente. No sólo se entiende, entonces que la producción de sentido se realice por la confluencia o convergencia de diversos referentes (lo cual también es el caso), sino que ninguno de ellos es monolítico, 
monosémico ni definitivo, sino susceptible siempre de distintas interpretaciones, diacrónica y sincrónicamente. Lo que, entonces, se convierte en objetivo de investigación en los ER es el dar cuenta de las posibles combinaciones y/o 'negociaciones' entre diferentes elementos en los intercambios mediáticos para comprender la producción misma de sentido, las fortalezas interpretativas y las significaciones que de todo ello resulten.

Recordemos, siguiendo a Hall (1987:136-138), tres tipos de descodificaciones a que puede dar lectura de un texto periodístico. La primera sería la dominante-hegemónica, que es la que sigue la propuesta interpretativa del productor del discurso. La segunda es la negociada, en la que tenemos una interpretación mixta entre la dominante-hegemónica y la de oposición. La tercera es la de oposición, en la que se hace una interpretación en contra de la propuesta por el productor. En la sociosemiótica exoinmanentista (ABRIL 2007) se tiene muy en cuenta la apropiación que los sujetos hacen de los discursos mediáticos. Como hemos visto, un concepto que se toma de la sociología es el de agenciamiento, que es la capacidad que tienen los actores sociales de actuar más allá de las constricciones impuestas por la estructura social. Como señala Orozco (2006:23),

Los ER también asumen la 'capacidad de agencia' de los sujetos sociales como condición de posibilidad para la negociación de significados y la producción de sentido por parte de los sujetos sociales. No sólo el simple reconocimiento de que los miembros de las audiencias son activos, sino el hecho de que su actividad no es mera reacción a estímulos, sin que por eso sea siempre consciente tampoco, sino que obedece a patrones socioculturalmente establecidos, aprendidos y desarrollados a lo largo de sus vidas y de su particular historicidad con el medio en cuestión. Por eso los ER eluden el determinismo y reconocen la creatividad y la iniciativa personal de los sujetos en sus intercambios comunicacionales. Creatividad acotada no solamente en lo individual sino, en última instancia, en lo cultural, lo social, lo histórico y lo político.

Como afirma Ferguson (2007: 74), "La dinámica de la semiótica social se sostiene gracias a una interminable negociación de la relación entre una serie de mensajes y discursos y las consecuencias y correlatos de esos discursos."

Como puede apreciarse los conceptos fundamentales de esta sociosemiótica giran en torno a cómo la persona inserta en un contexto histórico, social, cultural y personal determinado se apropia, en situaciones concretas, de los relatos mediáticos construyendo su propio discurso a partir de diferentes interacciones intertextuales y extratextuales.

En esta misma línea, Ferguson (2007: 74) afirma: "En todo análisis de semiótica social y del discurso hay - y debe haber - cierta 
dinámica. Esa dinámica tiene que abarcar la contradicción en los significados y también la presencia de significados contradictorios, a veces en el mismo texto. $Y$ es precisamente esa dinámica la que permite que el lector o el analista aborden un texto en un nivel que trascienda la mera identificación formal con sus características. El significado fue, es y será un campo polémico".

Así pues, de acuerdo con estas ideas, la sociosemiótica del periodismo pretende dar cuenta, por ejemplo en el caso de la producción de discursos por los medios de comunicación, de la organización del trabajo comunicativo, de la incidencia de las industrias comunicativas en la misma y de las dependencias políticas y económicas que condicionan toda industria de la comunicación; la sociosemiótica debe ir más allá del análisis exclusivamente textual. Incluso por lo que hace referencia a la lectura, como apunta Barry Jordan (1986: 48): "El hecho de tomar en cuenta el fenómeno de la intertextualidad sugiere que el objeto de análisis no es simplemente el texto, ni necesariamente el campo de conocimientos públicos con los que el texto se relaciona, sino algo bastante más sutil y complejo que tiene mucho que ver con la biografía y ambiente social del lector, esto es lo que pone en marcha aquella combinación concreta de elementos que funcionan juntos en la lectura". Es necesario el paso del lector modelo a los lectores empíricos (Rodrigo, 1995: 90-97), el estudio de las audiencias, el análisis de los efectos de los medios, etc. Por ello se hace imprescindible que la sociosemiótica del periodismo sea pluridisciplinar.

De hecho, desde la propia Escuela Semiótica de Paris, Claude Chabrol apuntaba a una "psico-socio-semiótica" de carácter pluridisciplinar. Chabrol (1982: 180) consideraba que no se trata de una simple colaboración entre dos disciplinas: “(...) Hace falta crear un espacio teórico nuevo, homogéneo con su propio principio de pertinencia, su generalidad específica, el de una psico-socio-semiótica discursiva". Por mi parte, no tengo tan claro si hay que crear un espacio propio o si la sociosemiótica debe reivindicar su carácter transversal, trashumante y mestizo. En lo que si estamos de acuerdo con Jordan (1986: 52) es que "Es muy difícil, por ejemplo, analizar los problemas de la producción del texto, de las lecturas del texto o hacer la historia social de sus distintas activaciones mediante el texto aislado, en sí mismo". Para estudiar la producción, la circulación y el consumo de los discursos de los medios de comunicación es necesario algo más que el análisis textual. Aunque también es posible hacer el estudio semiótico de una parte del proceso, por ejemplo de la producción periodística (RODRIGO, 
1995: 151-156). De todos modos, para la sociosemiótica, como afirma Ferguson (2007: 14), "Todo estudio de los medios está inextricablemente ligado al contexto social de su producción y utilización. Esto significa que estudiar los medios implica estudiar mucho más que los medios".

\section{REFERÊNCIAS}

ABRIL, G. Análisis crítico de textos visuales. Mirar lo que nos mira. Madrid: Síntesis, 2007.

ALVIRA MARTIN, F. "Perspectiva cualitativa-perspectiva cuantitativa en la metodología sociología", Revista Española de Investigación Sociológica, n²2, abril-junio, 1983, pp. 53-75.

BELTRÁN, Miguel "Cinco vías de acceso a la realidad social”, en M. García Ferrando, J. Ibañez y F. Alvira (comps) El análisis de la realidad social. Métodos y técnicas de investigación. Madrid: Alianza, 1989, pp.17-47.

CHABROL, C. "Les discours du pouvoir. Pour une psycho-sociosémiotique", en Jean Claude Coquet (ed.) Sémiotique.L'École de Paris. Paris: Hachette, 1982, pp.173-198.

DEL RINCÓN, D. et al. Técnicas de investigación en ciencias sociales. Madrid: Dykinson, 1995.

FERGUSON, R. Los medios bajo sospecha. Ideología y poder en los medios de comunicación. Barcelona:Gedisa, 2007 .

FOUCAULT, M. La arqueología del saber. México: Siglo XX, 1978.

GEERTZ, C. "Significación y accion social. La ideología como sistema cultural", en AA.VV. El proceso ideológico. Buenos Aires: Tiempo Contemporáneo, 1976, pp. 13-46.

GLASGOW Media Group Bad News. Londres: Routledge, 1977.

GLASGOW Media Group. More Bad News. Londres: Routledge, 1980.

GREIMAS, A.J. Maupassant. La sémiotique du texte. Paris: Seuil, 1976a.

Sémiotique et sciences sociales. Paris: Seuil, 1976b.

GREIMAS, A.J. y Courtés, J.Semiótica. Diccionario razonado de la teoría del lenguaje. Madrid: Gredo, 1982).

GREIMAS, A.J. y Courtés, J. Sémiotique. Dictionnaire raisonné de la théorie du langage II. Paris: Hachette, 1986. 
HALL, S. "Encoding/decoding", en Stuart Hall, S. et al. (eds.) Culture, Media, Language. Londres: Hutchinson, 1987, pp.128-138.

HODGE, R. y Kress, G. Social Semiotics. Cambridge: Polity PresS, 1991.

JORDAN, B. "Textos, contextos y procesos sociales", en Estudios Semióticos no 9, 1986, pp. 37-58.

NEUMAN, W.L. Social Research Methods. Qualitative and Quantitative Approaches. Needham Heights (Massachusetts): Allyn and Bacon, 1994.

OROZCO GÓMEZ, G. La Investigación en Comunicación desde la Perspectiva Cualitativa. La Plata (Argentina): Ediciones de Periodismo y Comunicación, 1996.

OROZCO GÓMEZ, G. Televisión, audiencias y educación. Buenos Aires: Norma, 2001.

OROZCO GÓMEZ, G. "Los estudios de recepción: de un modo de investigar a una moda, y de ahí a muchos modos", en Florencia Saintout y Natalia Ferrante (comp.) ¿Y la recepción? Balance crítico de los estudios sobre el público. Buenos Aires: La Crujía, 2006, pp.14-30.

RODRIGO ALSINA, M. Los modelos de la comunicación. Madrid: Tecnos, 1995, 2ª edición.

Teorías de la comunicación: ámbitos, métodos y perspectivas. Bellaterra (Barcelona): Universitat Autònoma de Barcelona, Servei de Publicacion, 2001.

Paidós, 2005.

La construcción de la noticia. Barcelona:

VAN DIJK, T. A. La noticia como discurso. Comprensión, estructura y producción de la información. Barcelona: Paidós 1990.

VERÓN, E. La semiosis social. Barcelona: Gedisa, 1987.

MIQUEL RODRIGO ALSINA é professor da Universidade Pompeu Fabra, na Espanha, e doutor pela Universidade Autônoma de Barcelona (UBA).

ARTIGO ORIGINALMENTE PUBLICADO EM JUNHO DE 2008. 